\section{Percepções de gestores, profissionais e usuários acerca do registro eletrônico de saúde e de aspectos facilitadores e barreiras para a sua implementação}

\author{
Views of health system administrators, \\ professionals, and users concerning the \\ electronic health record and facilitators and \\ obstacles to its implementation
}

\author{
Percepciones de gestores, profesionales y \\ usuarios acerca del registro electrónico de \\ salud y de aspectos facilitadores y barreras \\ para su implementación
}

Jose Felipe Riani Costa 1

Margareth Crisóstomo Portela 2

\title{
Resumo
}

A concepção e a implementação de tecnologias complexas, como o registro eletrônico de saúde (RES), envolvem tanto aspectos técnicos quanto questões pessoais, sociais e organizacionais. Há no cenário brasileiro público e privado diferentes iniciativas locais e regionais de implementação de RES. Existe ainda, por parte do Ministério da Saúde, a proposta de conformação de um RES nacional. O estudo aqui apresentado buscou propiciar uma visão abrangente das percepções de gestores, profissionais e usuários do sistema de saúde acerca de suas experiências com o RES e suas opiniões acerca da perspectiva de conformação de um RES nacional. Trata-se de um estudo qualitativo que envolveu a realização de 28 entrevistas semiestruturadas. Os resultados obtidos explicitaram, além da diversidade de fatores que podem influenciar a implementação do RES, a existência de confluências e de aspectos que tendem a ser valorizados de modo distinto, conforme os diferentes pontos de vista. Dentre os aspectos chave destacados nas percepções dos entrevistados, vale enfatizar as discussões acerca de atributos e do impacto do RES no cuidado de saúde, especialmente no caso dos RES de uso local; as preocupações com os custos e com o sigilo e privacidade, relativos aos RES em geral; e as possíveis implicações decorrentes da opção pela centralização ou descentralização do armazenamento das informações, no caso do RES nacional. Ficou patente nas falas dos entrevistados a necessidade de que seja estabelecida uma comunicação mais efetiva entre as partes interessadas, e que as diferentes perspectivas sejam consideradas na formulação e implementação de RES nos níveis local, regional e nacional.

Registros Eletrônicos de Saúde; Informática em Saúde Pública;

Comunicação em Saúde

\author{
Correspondência \\ J. F. R. Costa \\ Agência Nacional de Saúde Suplementar. \\ Av. Augusto Severo 84, Rio de Janeiro, RJ 20021-040, Brasil. \\ jfeliperiani@gmail.com \\ 1 Agência Nacional de Saúde Suplementar, Rio de Janeiro, \\ Brasil. \\ 2 Escola Nacional de Saúde Pública Sergio Arouca, Fundação \\ Oswaldo Cruz, Rio de Janeiro, Brasil.
}




\section{Introdução}

O registro eletrônico de saúde (RES) pode constituir uma base de informações em saúde individualizada segura, legível, consistente e relevante, mas também pode acarretar consequências não intencionais indesejáveis, como ameaças à segurança do paciente ou a insatisfação dos profissionais de saúde 1. Ainda que autores e instituições diferenciem o RES do prontuário eletrônico do paciente (PEP), neste artigo opta-se pelo uso do primeiro para identificar as diferentes experiências de informatização do registro, armazenamento e disponibilização de informações individualizadas de saúde.

Os RES são intervenções complexas, cuja implementação depende de uma intensa mobilização de recursos financeiros, tecnológicos e humanos 1,2 . Na perspectiva de imprimir caráter mais sistemático na implementação de intervenções complexas, autores têm incorporado abordagens da Ciência da Melhoria (Improvement Science), Ciência da Implementação e Pesquisa Translacional 3. Dentre eles, Perez et al. 4, desenvolveram um estudo sobre inovações tecnológicas na saúde sob a ótica da teoria de difusão, e Richardson et al. 1, exploraram o Consolidated Framework for Implementation Research (CFIR) como referencial teórico para avaliar qualitativamente a implementação de RES.

Potenciais utilizadores dos RES (gestores, profissionais e usuários do sistema de saúde) possuem um valioso conhecimento que pode incidir diretamente no sucesso ou insucesso da implementação 5,6. Contudo, apesar da complexidade e diversidade de aspectos envolvidos, identifica-se uma pequena proporção de estudos abrangendo perspectivas de diferentes partes interessadas 4,7,8 e a predominância de trabalhos que se concentraram em explorar as experiências locais com RES e as opiniões dos profissionais de saúde 5,9,10,11,12,13,14.

O estudo aqui apresentado teve como objetivo geral propiciar uma visão abrangente das percepções de gestores, profissionais e usuários do sistema de saúde acerca de suas experiências com RES e suas opiniões sobre a proposta do Ministério da Saúde de conformação de um RES nacional, de modo a oferecer subsídios para o planejamento e a implementação de RES no país.

Os objetivos específicos do trabalho foram: (1) apreender percepções relacionadas às experiências locais e regionais com o uso de RES; (2) explorar opiniões sobre o papel e a pertinência dos RES no cuidado de saúde; (3) captar visões acerca da proposta do Ministério da Saúde de conformação de um RES nacional; e (4) sistematizar aspectos facilitadores e barreiras percebidos pelos entrevistados para o desenvolvimento e incorporação dos RES no Brasil.

\section{Métodos}

Trata-se de um estudo qualitativo, que envolveu a realização de entrevistas individualizadas baseadas em roteiro semiestruturado. As entrevistas foram desenvolvidas com o propósito de explorar e compreender as percepções dos entrevistados sobre os RES, expressas com base em suas experiências pessoais e em processos sociais compartilhados 15 .

Foi utilizado um único modelo de roteiro semiestruturado para todos os entrevistados. Buscou-se usar palavras e expressões que pudessem ser compreensíveis por entrevistados de diferentes perfis e que permitissem a abordagem dos tópicos com base em diferentes pontos de vista. O roteiro de entrevistas foi pensando para cobrir os seguintes aspectos: (1) experiências prévias e expectativas dos entrevistados com RES; (2) possíveis vantagens dos RES; (3) possíveis desvantagens, riscos ou prejuízos associados aos RES; (4) opiniões dos entrevistados sobre um RES nacional; (5) barreiras e facilitadores para a implementação de RES; e (6) sugestões dos entrevistados sobre o que precisaria ser feito para a conformação de um RES nacional.

O total de entrevistas foi definido baseando-se no alcance da saturação teórica. A saturação teórica refere-se ao fato de a entrada de dados de novas entrevistas não acarretar a identificação de novas categorias de análise. As entrevistas foram gravadas digitalmente e transcritas na íntegra. A análise das transcrições foi realizada com o auxílio do software Nvivo, versão 10 (QSR International Pty, Doncaster, Austrália).

Há diversos aportes teóricos originários tanto do setor saúde quanto de fora dele que podem favorecer a compreensão dos fatores que influenciam a implementação de RES. Na presente pesquisa, buscou-se estudar e incorporar elementos de diferentes aportes teóricos da área de informação 
e informática, bem como de modelos desenvolvidos para a implementação de intervenções para a melhoria do cuidado de saúde.

Dentre os aportes teóricos estudados, o CFIR foi aquele que apresentou mais elementos que foram incorporados na pesquisa, sobretudo no processo de definição das categorias de análise. O CFIR, que representa um dos diferentes modelos propostos no âmbito da ciência da melhoria da qualidade do cuidado de saúde, foi elaborado por Damschroder et al. 16 com base na revisão de mais de 500 fontes publicadas relacionadas a 13 diferentes disciplinas científicas, tendo como uma das principais inspirações a teoria de difusão de inovações de Rogers 17.

Categorias de análise foram definidas originalmente com base no CFIR e refinadas no decorrer do processo de codificação das entrevistas para o melhor ajuste a dados captados. A Figura 1 sistematiza as categorias e subcategorias finais.

A seleção de entrevistados teve o propósito de abranger diferentes perfis e vivências, incluindo pessoas envolvidas em iniciativas incipientes de uso local e regional de RES, mas também experiências tidas como mais consolidadas. Os entrevistados foram distribuídos de acordo com as seguintes categorias: gestores, profissionais de saúde, profissionais de informática em saúde, profissionais de direito e ética, e usuários do sistema de saúde. Na categoria de gestores, foram considerados ainda o seu local de atuação, incluindo gestor de serviço de saúde, de operadora de planos privados de saúde e das administrações municipal, estadual e federal da saúde.

\section{Figura 1}

Categorias de análise do estudo.

\begin{tabular}{|c|c|}
\hline Características dos RES & $\begin{array}{l}\text { - Funcionalidades e conteúdo } \\
\text { - Usabilidade } \\
\text { - Precisão } \\
\text { > Funcionamento dos RES e integridade das informações } \\
\text { - Interoperabilidade } \\
\text { > Custo }\end{array}$ \\
\hline Fatores relacionados aos indivíduos & $\begin{array}{l}\text { C Conhecimentos e habilidades dos profissionais de saúde com a tecnologia } \\
\text { - Expectativas de resultados } \\
\text { - Resistência à mudança } \\
\text { - Aceitabilidade da informatização na saúde }\end{array}$ \\
\hline $\begin{array}{l}\text { Características das organizações de saúde e } \\
\text { cenários locais }\end{array}$ & $\begin{array}{l}\text { - Cultura organizacional e compatibilidade com processos de trabalho } \\
\text { Disponibilidade de recursos }\end{array}$ \\
\hline $\begin{array}{l}\text { Fatores externos à organização de saúde e macro } \\
\text { contexto }\end{array}$ & $\begin{array}{l}\text { Pressão mimética } \\
\text { - Aspectos éticos e legais } \\
\text { - Adoção de padrões } \\
\text { - Apoio técnico, financeiro e pressão coercitiva } \\
\text { > Antecedentes e outras experiências de informação e informática }\end{array}$ \\
\hline Processo & $\begin{array}{l}\text { > Planejamento } \\
\text { - Engajamento } \\
\text { - Execução } \\
\text { > Reflexão e avaliação }\end{array}$ \\
\hline \multicolumn{2}{|r|}{ Possibilidades técnicas do RES nacional } \\
\hline
\end{tabular}

RES: registro eletrônico de saúde. 


\section{Resultados e discussão}

Foram realizadas 28 entrevistas no total, distribuídas, segundo tipo de entrevistado, conforme a Tabela 1.

Dentre os dez gestores entrevistados, dois atuavam em secretarias de saúde de âmbito municipal e um em secretaria estadual; um em estabelecimento hospitalar; quatro no Ministério da Saúde; um na Agência Nacional de Saúde Suplementar; e um em operadora de planos privados de saúde.

Os profissionais de saúde entrevistados eram todos atuantes na prestação do cuidado, conforme pré-requisito estabelecido. Quatro atuavam em estabelecimentos públicos de saúde, dentro os quais, três na atenção básica e um em hospital. O quinto profissional de saúde entrevistado atuava no ambulatório de uma operadora de planos de saúde e em consultório próprio.

Dentre os cinco profissionais de informática em saúde entrevistados, quatro fizeram a graduação na área de exatas, com dois atuando no ambiente acadêmico, um em uma empresa desenvolvedora de software e um em operadora de planos privados de saúde. O quinto profissional fez graduação na área da saúde e participava de iniciativas no setor público e no setor privado suplementar de saúde.

Dos três profissionais de direito e ética entrevistados, um atuava no ambiente acadêmico e dois em ministérios públicos estaduais.

Dentre os cinco usuários entrevistados, um não era vinculado a nenhuma entidade representativa de usuários do sistema de saúde, dois eram vinculados a entidades representativas de usuários e os outros dois, além de vinculados a entidades representativas, participavam do Conselho Nacional de Saúde.

As entrevistas somaram, no total, pouco mais de 21 horas de áudio gravadas digitalmente, o que corresponde ao tempo médio de, aproximadamente, 45 minutos por entrevista.

Na Figura 2 é apresentada uma síntese dos resultados, com as principais barreiras e aspectos facilitadores para a implementação de RES percebidos pelos entrevistados.

A maioria das barreiras e aspectos facilitadores identificados se relaciona aos RES de modo geral, sejam eles de âmbito local, regional ou nacional. A relação entre as principais barreiras e aspectos facilitadores aos RES e o fato de alguns deles incidirem de modo mais específico sobre a proposta do RES nacional são explorados a seguir.

Dentre os aspectos chave destacados nas percepções dos entrevistados, vale enfatizar as discussões acerca de atributos e do impacto dos RES no cuidado de saúde, especialmente no caso dos RES de uso local; as preocupações com os custos e com o sigilo e privacidade, relativos aos RES em geral; e as possíveis implicações decorrentes da opção pela centralização ou descentralização do armazenamento das informações, no caso do RES nacional.

Tabela 1

Total de entrevistas realizadas, por tipo de entrevistado.

\begin{tabular}{lc}
\hline Entrevistados & $\mathbf{n}$ \\
\hline Gestores & 10 \\
Profissionais de saúde & 5 \\
Profissionais de informática em saúde & 5 \\
Usuários do sistema de saúde & 5 \\
Profissionais de direito e ética & 3 \\
Total & 28 \\
\hline
\end{tabular}


Figura 2

Síntese das barreiras e aspectos facilitadores percebidos.

\begin{tabular}{|c|c|c|}
\hline Categoria & Barreiras & Facilitadores \\
\hline Características dos RES & $\begin{array}{l}\text { - Informações redundantes } \\
\text { - Excesso de informações para preencher } \\
\text { - Dificuldade para acessar informações registradas e } \\
\text { para emitir relatórios } \\
\text { > Lentidão no processamento } \\
\text { > Instabilidade e travamento do sistema } \\
\text { - Defeitos técnicos que alteram a informação } \\
\text { registrada } \\
\text { > Possibilidade de perder a informação registrada } \\
\text { > Custo para desenvolver ou adquirir RES } \\
\text { > Custo para manter RES }\end{array}$ & $\begin{array}{l}\text { - Funcionalidades e conteúdos úteis } \\
\text { - Integração de informações registradas em diferentes } \\
\text { momentos, por diferentes pessoas } \\
\text { > Facilidade de acesso às informações } \\
\text { > Redução nas omissões, confusões e equívocos nas } \\
\text { informações repassadas pelos pacientes } \\
\text { - Melhor compreensão, evitando problemas com a } \\
\text { caligrafia dos profissionais de saúde } \\
\text { > Tendência de diminuição dos custos dos RES } \\
\text { > Relação custo/efetividade } \\
\text { > Opção por tecnologias de baixo custo }\end{array}$ \\
\hline $\begin{array}{l}\text { Fatores relacionados aos } \\
\text { indivíduos }\end{array}$ & $\begin{array}{l}\text { Falta de conhecimentos e habilidades dos } \\
\text { profissionais de saúde com tecnologia } \\
\text { Pessoas com baixa instrução ou com mais idade } \\
\text { tendem a ter mais dificuldade com a tecnologia } \\
\text { - Possibilidade de tornar o atendimento mais } \\
\text { demorado } \\
\text { - Resistência à mudança } \\
\text { - Resistência dos profissionais de saúde em função do } \\
\text { constrangimento à autonomia } \\
\text { salta de comprometimento dos profissionais de } \\
\text { saúde com a qualidade da informação } \\
\text { existentes }\end{array}$ & $\begin{array}{l}\text { Tendências das pessoas terem cada vez mais } \\
\text { familiaridade com as tecnologias } \\
\text { - Entendimento de que a informatização é algo natural } \\
\text { Possibilidade de agilizar o atendimento } \\
\text { na mendência de que a resistência inicial seja superada } \\
\text { dos RES } \\
\text { - Favorecimento da transparência percebidas as utilidades } \\
\text { - Oportunidade de aperfeiçoar os processos de } \\
\text { trabalho } \\
\text { - Estímulo ao trabalho colaborativo da equipe de } \\
\text { saúde } \\
\text { - Expectativas positivas para a gestão em saúde, em } \\
\text { especial o acesso a informações relevantes sobre a } \\
\text { atenção à saúde e seus resultados } \\
\text { - Expectativas positivas para o trabalho em saúde, } \\
\text { em especial à continuidade do cuidado e o suporte à } \\
\text { decisão } \\
\text { - Expectativas positivas para o usuário do sistema de } \\
\text { saúde, em especial o autocuidado e o controle social }\end{array}$ \\
\hline $\begin{array}{l}\text { Características das } \\
\text { organizações de saúde e } \\
\text { cenários locais }\end{array}$ & $\begin{array}{l}\text { - Cultura organizacional desfavorável à mudança } \\
\text { - Cultura organizacional desfavorável à informatização } \\
\text { - Falta de priorização da informática em saúde } \\
\text { - Falta de investimentos financeiros } \\
\text { - Problemas com rede de Internet } \\
\text { - Computadores antigos e falta de computadores } \\
\text { - Falta de pessoas capacitadas em informática em } \\
\text { saúde } \\
\text { - Fragilidade política, técnica, econômica e financeira } \\
\text { de municípios }\end{array}$ & $\begin{array}{l}\text { > Cultura organizacional favorável à mudança } \\
\text { > Cultura organizacional favorável à informatização } \\
\text { > Investimentos financeiros em informática em saúde }\end{array}$ \\
\hline
\end{tabular}

(continua) 


\begin{tabular}{|c|c|c|}
\hline Categoria & Barreiras & Facilitadores \\
\hline $\begin{array}{l}\text { Fatores externos à } \\
\text { organização de saúde e } \\
\text { macro contexto }\end{array}$ & $\begin{array}{l}\text { - Frustração e descrédito decorrentes de fracassos em } \\
\text { experiências anteriores } \\
\text { - Risco do uso indevido da informação } \\
\text { > Receio de acessos não autorizados } \\
\text { > Falta de integração entre os Sistema de Informação } \\
\text { em Saúde existentes } \\
\text { > Limitações e dificuldade de uso das soluções de } \\
\text { informática providas pelo Ministério da Saúde } \\
\text { > Padrões para interoperabilidade sintática e } \\
\text { semântica ainda não consolidados } \\
\text { - Falta de legislação específica sobre privacidade das } \\
\text { informações e sobre consentimento informado dos } \\
\text { usuários do sistema de saúde }\end{array}$ & $\begin{array}{l}\text { - Acúmulos, avanços e aprendizados com experiências } \\
\text { anteriores } \\
\text { > Compreensão de que a existência de eventuais riscos } \\
\text { à privacidade não deve impedir o uso de RES } \\
\text { > Possibilidade técnica de aperfeiçoar o sigilo da } \\
\text { informação eletrônica } \\
\text { > Tradição do país com sistema de informação em } \\
\text { saúde de base nacional } \\
\text { > Iniciativas do Ministério da Saúde de prover soluções } \\
\text { de informática } \\
\text { > Avanços na adoção de padrões de interoperabilidade } \\
\text { sintática e semântica }\end{array}$ \\
\hline Processo & $\begin{array}{l}\text { - Complexidade sociotécnica envolvida na } \\
\text { implementação } \\
\text { - Falta de diálogo entre os gestores e as demais partes } \\
\text { envolvidas } \\
\text { Falta de treinamento e de capacitação } \\
\text { - Poucas oportunidades para os profissionais de saúde } \\
\text { participarem do planejamento, desenvolvimento, } \\
\text { escolha e manutenção dos RES } \\
\text { - Falta de cumprimento dos documentos e } \\
\text { instrumentos formais } \\
\text { - Descontinuidades e retrocessos no decorrer dos } \\
\text { processos } \\
\text { - Disputas decorrentes de interesses políticos e } \\
\text { econômicos }\end{array}$ & $\begin{array}{l}\text { - Realização de piloto e adoção de estratégias } \\
\text { incrementais } \\
\text { > Ampliação da divulgação e do diálogo com a } \\
\text { sociedade } \\
\text { > Realização de treinamentos e capacitações buscando } \\
\text { orientar e motivar as partes interessadas } \\
\text { > Identificação de "campeões" de projeto } \\
\text { - Elaboração e publicação de documentos e } \\
\text { instrumentos formais } \\
\text { > Feedback sobre o processo às pessoas e equipes }\end{array}$ \\
\hline
\end{tabular}

RES: registro eletrônico de saúde.

\section{Atributos e impacto do RES no cuidado de saúde}

No âmbito do presente estudo, os entrevistados foram estimulados, na parte inicial das entrevistas, a discorrer sobre as principais funcionalidades que acreditavam que os RES deveriam ter. Isso porque a menção espontânea a determinadas funcionalidades pode denotar, em certa medida, o valor dado pelos entrevistados às diferentes funcionalidades.

Cabe destacar a ênfase dada por diferentes entrevistados à perspectiva de que a continuidade do cuidado e o suporte à decisão podem ser favorecidos com base nos conteúdos e funcionalidades vinculados aos RES. Por exemplo, um médico entrevistado mencionou que, com o uso do registro, “(...) um profissional de saúde tem acesso ao prontuário do outro. Então (...) eu tenho como entrar no prontuário do cardiologista e verificar como foi a consulta, a queixa, conduta, exames, enfim, e isso é muito bom (...)”. Um profissional de informática em saúde destacou a possibilidade de "incorporar sistemas de apoio à decisão, críticas dos mais diversos tipos”. Observou-se que a maior parte das menções se restringiu às funcionalidades básicas dos RES, o que pode ser reflexo do estágio ainda incipiente de experiências de RES no país.

Além da utilidade percebida, a intenção que as pessoas têm de usar ou não um sistema de informação está intimamente associada à percepção delas sobre a facilidade de uso 4,18,19. A imperatividade de que os RES sejam de fácil utilização e tenham utilidade prática foi manifestada por diferentes 
entrevistados, em especial por profissionais de saúde. Em convergência com o observado no presente trabalho, a revisão da literatura sobre experiências de implementação de RES em países desenvolvidos, conduzida por McGinn et al. 5 , apontou que as preocupações técnicas com o funcionamento, com a facilidade de uso e com a utilidade dos RES estiveram entre os fatores destacados por profissionais de saúde.

A tendência de o profissional de saúde enfatizar questões relacionadas à facilidade de uso e à utilidade dos RES pode estar associada aos possíveis impactos diretos na sua rotina de trabalho. Neste estudo, foram identificados relatos de entrevistados sobre diferentes problemas com RES de uso local, como lentidão no processamento, instabilidade, perda de informações registradas, excesso de informações para preencher, informações repetidas e dificuldades para consultar as informações registradas. A fala mais emblemática nesse sentido foi a da médica de um centro de atenção básica de saúde, que se recusou a usar o RES adotado no estabelecimento, alegando falhas na concepção do sistema e dificuldades de uso.

“(...) tenho que repetir digitação de data de nascimento, de nome da mãe..., eu sou médica, eu odeio fazer esta tarefa, odeio! Eu não suporto, eu gosto de saber assim: 'Qual o seu problema? O que traz você aqui?'”

"Eu mesma, não estou usando esse prontuário eletrônico porque eu não consegui ver essa agilidade nele, entendeu? Só de preencher item por item, ficar preenchendo ali, depois ele vai num outro profissional, tem que preencher os mesmos itens (...)".

No que se refere ao impacto dos RES no tempo de atendimento em saúde, houve opiniões de que, a depender das características dos registros, dos computadores e da rede de internet, a adoção de RES pode colaborar tanto para tornar o atendimento mais rápido quanto contribuir para atrasos e aumentar a carga de trabalho dos profissionais de saúde. Um dos profissionais de saúde entrevistados referiu que com o registro "Ganha tempo, tem a informação mais selecionada e mais fácil de você encontrar", aspecto referido em estudos nacionais e internacionais 7,10,20.

Por outro lado, houve entrevistados que mencionaram a possibilidade de os RES demandarem mais tempo do profissional de saúde no preenchimento, por exigirem a entrada de dados em maior quantidade ou de forma mais estruturada, em convergência com Silva et al. 21. A referência a uma fase inicial de adaptação foi verificada na fala de alguns dos entrevistados, tal como identificado por Wechsler et al. 22.

No que se refere à relação entre o profissional de saúde e o paciente, há estudos que 23 enfatizam a importância de que a alta tecnologia não seja superestimada e acabe por comprometer a humanização do cuidado 9,23,24,25. Porém, há também trabalhos que identificam opiniões de que o uso do RES não apresentaria interferência na relação entre o profissional de saúde e o paciente ou, ainda, que o registro poderia melhorar a referida relação 22,26,27. Nesta pesquisa, foram identificadas percepções de entrevistados no sentido de que a relação entre profissional de saúde e paciente pode melhorar com o uso do RES, na medida em que informações relevantes sejam disponibilizadas de forma organizada, reduzindo omissões, confusões e equívocos.

No entendimento de profissionais de saúde entrevistados, parte dos problemas com os RES poderia ser evitada se as opiniões deles fossem consideradas no planejamento e nas etapas relativas ao desenvolvimento, aquisição ou adaptação dos sistemas. Nesse sentido, um profissional de saúde entrevistado disse que:

"Se um presidente, um diretor, um gestor quer implantar um sistema, é preciso conversar com as pessoas que vão usar esse sistema, os profissionais de saúde, com os profissionais do arquivo que precisam se familiarizar com essa tecnologia (...) não adianta vir de cima pra baixo, né? Ele tem que conversar com quem vai usar para saber qual o melhor sistema né? (...). Não adianta eu chegar para quem tá na ponta e dizer: 'A partir de hoje você vai fazer isso"'.

O fato de uma intervenção ser desenvolvida externamente, associada à falta de transparência no processo de tomada de decisão e a uma adaptação ineficaz, aumenta a probabilidade de insucesso de sua implementação 28 . Autores apontam que é essencial que as pessoas que utilizam os RES participem das diferentes etapas relacionadas ao planejamento e à implementação 29,30.

Já na opinião de alguns dos gestores entrevistados, parte das reclamações de profissionais de saúde sobre problemas com os registros podem ser subterfúgio para encobrir resistências relacionadas ao receio de perda de autonomia, à falta de habilidade com informática ou ao descompromisso com a qualidade da informação em saúde. 
O fato de parte dos profissionais de saúde ter receio de perder autonomia com o uso de RES foi apontado por gestores, profissionais de saúde, profissionais de informática em saúde e usuários entrevistados. Um dos usuários entrevistados, por exemplo, disse que o RES:

“(...) é uma forma de controle do gestor, e o profissional não gosta de ser controlado, né? Ele gosta de ter autonomia. Ele acha que a avaliação não deve ser feita, mas se está tudo escrito ali, o gestor tem como ver se o cara está cumprindo o que ele deveria fazer (...) então eu acho que o profissional pode se ressentir por causa disso (...)”.

A compreensão dos profissionais de saúde de que determinada intervenção possa representar uma ameaça à sua autonomia tem forte influência no fato de eles "abraçarem" ou não uma intervenção e pode representar uma barreira à sua implementação 4 . O fato de uma pessoa ter alguma resistência prévia a uma tecnologia da informação pode enviesar a sua percepção sobre a utilidade da tecnologia e interferir na sua intenção de utilizá-la 31.

Uma gestora entrevistada, por exemplo, entende que "para minimizarem a sua dificuldade, elas (as pessoas que utilizam os RES) acabam botando em cima do sistema a dificuldade”. A capacidade de um indivíduo utilizar uma intervenção está associada ao conhecimento prévio de como fazer o uso adequado. Se as pessoas não obtêm o conhecimento necessário sobre uma tecnologia antes de começarem a utilizá-la, são maiores as possibilidades de desistência e de rejeição 32 .

A necessidade de realização de treinamento e capacitação das pessoas para fazerem uso dos registros foi apontada tanto por gestores quanto por profissionais e usuários do sistema de saúde entrevistados. Houve profissionais de saúde entrevistados que descreveram agir como autodidatas e que descobriram o modo de utilizar os RES a partir de tentativa e erro e em conversas com outros profissionais de saúde.

No que se refere ao grau de comprometimento dos profissionais de saúde com a informação em saúde, o fato do uso de um sistema de informação não representar, tipicamente, a atividade-fim de um profissional da saúde tem forte influência nas resistências à sua aceitação 33. Uma das gestoras entrevistadas apontou que: "as pessoas que não entendem a dimensão do registro de saúde" e "fazem um cadastro do paciente de qualquer jeito".

Dentre as menções de entrevistados à existência de resistências à mudança, uma profissional de saúde, por exemplo, disse que:

“(...) toda novidade na verdade eu acho que gera, a princípio, um aspecto de resistência. Então, se um hospital ou uma unidade de saúde não tem um registro [eletrônico de saúde] e chega uma novidade que vai ser implantada, (...) tem que vencer essa resistência dos profissionais (...). A pessoa vai ter que ser motivada, treinada, isso tudo".

No entanto, houve também diferentes menções à possibilidade de que as resistências iniciais aos RES sejam vencidas com o tempo, na medida em que as pessoas entendam melhor o sistema e percebem vantagens. Uma gestora entrevistada comentou que, no "início”, a implantação do RES no hospital em que trabalhava "foi um caos", mas que "depois as pessoas veem que é tão bom, que elas nunca mais querem largar”. Se as pessoas identificam claramente a utilidade de uma intervenção e a possibilidade de que ela facilite o seu trabalho, é mais provável que a implantação seja bem-sucedida 11 .

\section{Custos, sigilo e privacidade}

Entre os aspectos recorrentes na fala dos entrevistados de diferentes perfis, vale destacar as preocupações com o custo e com o sigilo e a privacidade das informações armazenadas nos registros. Tal condição também foi apontada em revisão de McGinn et al. 6, na qual essas preocupações estiveram dentre as identificadas como comuns às diferentes partes envolvidas na implementação de RES.

A preocupação com o sigilo e a privacidade esteve presente, em maior ou menor grau, nas falas de todos os entrevistados neste estudo. Especificamente, entre os usuários do sistema de saúde entrevistados, notou-se que os dois que participavam do Conselho Nacional de Saúde deram maior destaque à questão do sigilo e da privacidade.

"A gente sempre se preocupou com a segurança nesse item. A gente debateu longamente. (...) isso é indispensável pra poder a coisa andar e, acima de tudo, beneficiar o usuário".

"Nos preocupa muito, acho que tem pouca confiança. Melhorou muito no Brasil, (...) mas eu, particularmente, não confio em muitas das informações que circulam eletronicamente. Eu ainda sou uma cidadã que bota um pé atrás". 
A responsabilidade pela representação formal dos usuários no Conselho Nacional de Saúde pode ter contribuído para a preocupação em se posicionar no sentido da legítima defesa dos interesses do conjunto de usuários do sistema de saúde no sigilo de suas informações de saúde. Ademais, a participação desses entrevistados em discussões sobre o assunto no Conselho Nacional de Saúde pode ter favorecido o acesso a conhecimentos técnicos relacionados a eventuais riscos de violação do sigilo de informações armazenadas eletronicamente. Assim, pode ser um indicativo da politização do debate sobre sigilo e privacidade ou da falta de informação para os usuários "comuns" acerca dos direitos e dos riscos envolvidos com as informações de saúde.

Ainda quanto ao sigilo das informações de saúde, diferentes entrevistados manifestaram a necessidade de aperfeiçoamento da regulamentação sobre o assunto. Nesse sentido, Freire 34 aponta para a necessidade de consolidação das várias normas em uma legislação específica para a saúde, e que sejam abordados aspectos não contemplados ou não definidos com clareza, como a questão do consentimento prévio dos pacientes sobre a coleta e uso de informações pessoais e o estabelecimento de penalidades efetivas por quebras de privacidade.

Sobre os custos, a preocupação com a necessidade de investimentos financeiros para se desenvolver ou adquirir um RES esteve presente na fala de diferentes entrevistados. Os custos associados à realização de treinamentos e à manutenção e evolução dos registros também foram mencionados. A crise financeira pela qual passa o país foi lembrada por alguns entrevistados como uma barreira ao RES, em especial na perspectiva de um RES de base nacional.

\section{Proposta do Ministério da Saúde de registro eletrônico nacional}

Especificamente quanto à aceitabilidade do RES nacional, dentre os entrevistados que se mostraram favoráveis à sua conformação, as principais motivações referidas foram as potenciais utilidades do RES no planejamento, programação, controle e avaliação, bem como a perspectiva de favorecer a continuidade do cuidado e a pesquisa. Tais vantagens, também associadas por entrevistados aos RES de âmbito local, foram destacadas por alguns entrevistados como passíveis de serem potencializadas com a conformação de um RES de alcance nacional.

Dentre os entrevistados que manifestaram ressalvas ou que julgaram não ser pertinente a conformação de um RES de base nacional, uma das principais motivações foi o entendimento de que o grau de complexidade da proposta pode inviabilizar a sua concretização. A falta de padrões de interoperabilidade, as desigualdades de infraestrutura e de recursos financeiros e humanos, o subfinanciamento da saúde e o fato de a informática em saúde não ser prioridade foram alguns dos fatores destacados por entrevistados dentre as barreiras para os RES no âmbito local e para a estruturação de um RES de base nacional.

Além dos aspectos referidos, parte das opiniões dos entrevistados sobre a proposta do registro nacional se concentrou na arquitetura de armazenamento das informações, com destaque para as seguintes possibilidades: arquitetura centralizada, com o armazenamento das informações em repositórios centralizados; ou de modo distribuído, com as informações armazenadas no âmbito dos computadores localizados nos estabelecimentos de saúde.

$\mathrm{Na}$ opinião dos entrevistados que entendem ser pertinente investir na arquitetura centralizada, foi expressa a possibilidade de que a utilização de uma estrutura de barramento de dados auxilie a validação e a qualificação das informações que vão para os repositórios centralizados. Outro aspecto mencionado foi a possibilidade do estabelecimento de mecanismos que confiram maior segurança no armazenamento dos dados. Um aspecto facilitador nesse sentido seria o fato de o Brasil ser um país com tradição no desenvolvimento de sistemas de base nacional e o fato de o Ministério da Saúde ter constituído uma infraestrutura de armazenamento de dados que, segundo um gestor entrevistado, seria suficiente para dar início a um RES nacional.

Contudo, foram identificadas opiniões de entrevistados no sentido de que o armazenamento das informações em repositórios centralizados representaria uma ameaça à privacidade e segurança, considerando que a eventual quebra do sigilo poderia desvelar um conjunto amplo e estratégico de informações, preocupação compartilhada também por autores como Campion-Awwad et al. 35 . Outro fator apontado por entrevistados como uma desvantagem dos repositórios centralizados seria o custo para a constituição de infraestrutura para o armazenamento das informações. Assim, na opinião 
de alguns dos entrevistados, seria mais vantajoso o investimento em estratégias descentralizadas, investindo na interoperabilidade entre sistemas, de modo que um RES de uso local possa "conversar" diretamente com outro.

Em contraposição à constituição de repositórios centralizados de informação, há autores que defendem a concepção de sistema distribuído. Segundo Pisa et al. 36, o sistema distribuído pode proporcionar a conexão direta entre os computadores, de modo que cada integrante da rede tenha a possibilidade de gerenciar as informações sob sua posse e de consultar as informações armazenadas nos demais participantes da rede. Desse modo, os computadores atuam, simultaneamente, como clientes e servidores da rede 36,37 .

No cenário de computadores potentes e redes rápidas, a arquitetura distribuída surge como uma maneira de integrar sistemas de informação escritos em diferentes linguagens de programação. Porém, para atingir o nível de integração necessário, são necessários avanços na padronização dos serviços de software e a conformação de serviços acessíveis a diferentes aplicações, disponibilizando interfaces padronizadas e permitindo a troca de informações entre os sistemas 38,39,40.

No que se refere às desvantagens apontadas por entrevistados para a arquitetura distribuída, a principal foi a necessidade de que os computadores e máquinas nos estabelecimentos de saúde em que as informações estejam armazenadas fiquem disponíveis em tempo integral.

Houve entrevistados que citaram o fato de o Ministério da Saúde considerar a conformação de uma arquitetura híbrida do armazenamento das informações, sendo parte centralizada e parte distribuída. Porém, tanto informações disponibilizadas pelo Ministério da Saúde 41,42 quanto a fala de entrevistados denotam que a opção inicial do Ministério da Saúde foi a de investir na arquitetura centralizada. Assim, ainda estaria em estudo a possibilidade de se trabalhar, de modo complementar, com uma arquitetura distribuída para o RES nacional.

De acordo com a proposta do Ministério da Saúde, será armazenado nos repositórios centralizados um subconjunto das informações captadas por outros sistemas de informações e RES existentes. A experiência inglesa 35 foi mencionada por diferentes entrevistados como uma fonte de aprendizado para a decisão de trabalhar com um conjunto sumário de informações no nível nacional. Isso porque a opção inicial do governo inglês, de abarcar um amplo conjunto de informações no RES de base nacional, foi revista diante da complexidade e custo envolvidos em sua consecução.

No entanto, existem também outras "lições" que podem ser apreendidas da experiência inglesa, como a resistência gerada quando as partes envolvidas entendem que suas perspectivas não foram devidamente consideradas 43 . Heeks 44 refere, no âmbito do Design Reality Gap Model, que o envolvimento das partes interessadas contribui para a diminuição da diferença entre a concepção de um sistema e a realidade prática, ampliando as possibilidades de sucesso na implementação de sistemas de informação em saúde. Não obstante a existência de iniciativas como a publicação da Política Nacional de Informação e Informática em Saúde (PNIIS) 45 e o processo de elaboração da Estratégia e-Saúde para o Brasil 46, é necessária a ampliação da discussão sobre a proposta em questão, o que parece ainda ser um caminho a ser percorrido, segundo falas ouvidas nas entrevistas.

\section{Considerações finais}

Ao explorar diferentes perspectivas e ao abordar aspectos de experiências locais, regionais e a proposta do Ministério da Saúde de um RES nacional, em contraste com uma maioria de estudos calcados somente na perspectiva de profissionais de saúde em experiências locais, este estudo possibilitou a conformação de um conjunto abrangente de resultados e permitiu algumas reflexões sobre as confluências e contrastes entre diferentes perspectivas. Essa trama de perspectivas e visões reflete a complexidade da implementação de RES, que perpassa por mudanças culturais e envolve recursos, relações de poder e interesses políticos e econômicos.

Por outro lado, vale destacar que a amostra não foi constituída de modo a ser generalizável e não tem, portanto, a pretensão de apresentar a visão dos "gestores" ou dos "profissionais de saúde", por exemplo, de uma experiência local e muito menos do país. A interpretação dos achados deve levar em consideração o fato de que as opiniões aqui apresentadas e discutidas se referem ao conjunto de indivíduos entrevistados. 
Os resultados evidenciam a perspectiva sociotécnica envolvida na implementação dos registros, abrangendo aspectos tecnológicos, pessoais, organizacionais e sociais.

Dois aspectos são aqui destacados. No âmbito dos RES de uso local, o contraste entre a perspectiva apresentada por profissionais de saúde e por gestores, com os primeiros enfatizando a necessidade de que os registros sejam de fácil uso e tenham utilidade prática, enquanto os últimos entendem que parte das reclamações de profissionais de saúde sobre os RES seria subterfúgio para encobrir dificuldades com informática e estaria relacionada à preocupação com a perda da autonomia e à pouca importância dada à informação em saúde.

No que se refere à possibilidade de estruturação de um RES de base nacional, a principal oposição entre as ideias expressas pelos entrevistados se referiu à conformação das bases de dados de modo centralizado versus o modo distribuído. Nesse debate, as manifestações de alguns dos entrevistados dão a entender que suas opiniões sobre a conformação do RES nacional podem refletir posições ideológicas e convicções de ordem mais geral. Nas falas de entrevistados, assim como na literatura, são mencionadas vantagens e desvantagens relacionadas à arquitetura centralizada ou distribuída do armazenamento das informações. Nota-se, porém, que a oposição entre essas duas configurações não foi expressa por alguns dos entrevistados como algo absoluto, de modo que as opções não foram consideradas necessariamente excludentes. Foi percebida, inclusive, alguma relutância por parte de alguns dos entrevistados em se posicionar de forma mais categórica sobre qual seria, na opinião deles, a melhor forma para o armazenamento dos dados no RES nacional.

Em termos gerais, tanto na perspectiva do impacto do uso dos RES nos serviços de saúde quanto da estruturação de um RES nacional, merecem destaque as menções à necessidade de que seja estabelecida uma comunicação mais efetiva entre as partes interessadas e que as diferentes perspectivas sejam consideradas na formulação e na implementação de RES.

\section{Colaboradores}

J. F. R. Costa trabalhou na pesquisa, análise e redação do artigo. M. C. Portela realizou a revisão crítica do texto e aprovação da versão final.

\section{Agradecimentos}

Agradecemos a todos que colaboraram com este artigo, em especial aos entrevistados no estudo.

\section{Referências}

1. Richardson JE, Abramson EL, Pfoh ER, Kaushal R. Bridging informatics and implementation science: evaluating a framework to assess electronic health record implementations in community settings. AMIA Annu Symp Proc 2012; 2012:770-8.

2. Greenhalgh T, Morris L, Wyatt JC, Thomas G, Gunning K. Introducing a nationally shared electronic patient record: case study comparison of Scotland, England, Wales and Northern Ireland. Int J Med Inform 2013; 82:e125-38.

3. Portela MC, Pronovost PJ, Woodcock T, Carter P, Dixon-Woods M. How to study improvement interventions: a brief overview of possible study types. BMJ Qual Saf 2015; 24:325-36.

4. Perez G, Zwicker R, Zilber MA, Medeiros Junior A. Adoção de inovações tecnológicas na área de saúde: um estudo sobre sistemas de informação sob a ótica da teoria de difusão. Journal of Information Systems and Technology Management 2010; 7:71-94. 
5. Terry AL, Stewart M, Fortin M, Wong ST, Kennedy M, Burge F, et al. Gaps in primary healthcare electronic medical record research and knowledge: findings of a pan-Canadian study. Healthc Policy 2014; 10:46-59.

6. McGinn CA, Grenier S, Duplantie J, Shaw N, Sicotte C, Mathieu L, et al. Comparison of user groups' perspectives of barriers and facilitators to implementing electronic health records: a systematic review. BMC Med 2011; 9:46.

7. Luchenski SA, Reed JE, Marston C, Papoutsi C, Majeed A, Bell D. Patient and public views on electronic health records and their uses in the United Kingdom: cross-sectional survey. J Med Internet Res 2013; 15:e160.

8. Stumpf MK, Freitas HMR. A gestão da informação em um hospital universitário: o processo de definição do Patient Core Record. Revista de Administração Contemporânea 1997; 1:71-99.

9. Mourão AD, Neves JTR. Impactos da implantação do prontuário eletrônico do paciente sobre o trabalho dos profissionais de saúde da Prefeitura Municipal de Belo Horizonte. http://www.aedb.br/seget/arquivos/ artigos07/56_SEGET.pdf (acessado em 10/ Ago/2014)

10. Joia LA, Magalhães C. Evidências empíricas da resistência à implantação de prescrição eletrônica: uma análise explano-exploratória. RAC -Eletrônica 2009; 3:81-104.

11. Perez G, Zwicker R. Fatores determinantes da adoção de sistemas de informação na área de saúde: um estudo sobre o prontuário médico eletrônico. Revista de Administração Mackenzie 2010; 11:174-200.

12. Godoy JSM, Gonçalves LS, Peres AM, Wolff LDG. O uso do prontuário eletrônico por enfermeiros em unidades básicas de saúde brasileiras. J Health Inform 2012; 4:3-9.

13. Poli AG. As compreensões que o prontuário eletrônico do paciente assume no coletivo de trabalhadores de uma Unidade Básica de Saúde. http://coleciona-sus.bvs.br/lildbi/docsonli ne/get.php?id=508 (acessado em 19/Jan/2015).

14. Namorato L, Cavalcanti Neto AJ, Garani FV, Braga PO, Lustosa SAS. A utilização do prontuário eletrônico do paciente por médicos do Hospital Municipal Dr. Munir Rafful: um estudo de caso. J Health Inform 2013; 5:39-43.

15. Deslandes SF, Gomes R, Minayo MCS. Pesquisa social: teoria, método e criatividade. 31a Ed. Petrópolis: Editora Vozes; 2012.

16. Damschroder LJ, Aron DC, Keith RE, Kirsh SR, Alexander JA, Lowery JC. Fostering implementation of health services research findings into practice: a consolidated framework for advancing implementation science. Implement Sci 2009; 4:50.

17. Rogers EM. Diffusion of innovations. 3rd Ed. New York: The Free Press; 1971.

18. De Lone WH, McLean ER. The De Lone and McLean Model of information systems success: a ten-year update. Journal of Management Information Systems 2003; 19:9-30.
19. Meyer AD, Goes JB. Organizational assimilation of innovations: a multilevel contextual analysis. Acad Manage J 1988; 31:897-923.

20. Lima DFB, Braga ALS, Fernandes JL, Brandão ES. Sistema de informação em saúde: conceções e perspectivas dos enfermeiros sobre o prontuário eletrônico do paciente. Referência 2011; serIII:113-9.

21. Silva VCG, Betta CA, Nishio EA, Barsotini CNG, Wainer J. Mensuração do tempo dos registros manual e eletrônico da Sistematização da Assistência de Enfermagem em Unidade de Terapia Intensiva. J Health Inform 2012; 4: $37-42$.

22. Wechsler R, Anção MS, Campos CJR, Sigulem D. A informática no consultório médico. J Pediatr (Rio J.) 2003; 79 Suppl 1:S3-12.

23. Marin HF, Cunha ICKO. Perspectivas atuais da informática em enfermagem. Rev Bras Enferm 2006; 59:354-7.

24. Russell SC, Spooner SA. Barriers to EMR adoption in internal medicine and pediatric outpatient practices. Tenn Med 2004; 97: 457-60.

25. Linder JA, Schnipper JL, Tsurikova R, Melnikas AJ, Volk LA, Middleton B. Barriers to electronic health record use during patient visits. AMIA Annu Symp Proc 2006; 2006:499-503.

26. David B, Ashley S. An Australian case study of patient attitudes towards the use of computerised medical records and unique identifiers. Stud Health Technol Inform 2001; 84(Pt 2):1430-4

27. Dagnone RV, Wilson R, Goldstein DH, Murdoch J, Rimmer MJ, VanDenKerkhof EG. How do patients perceive electronic documentation at the bedside? J Healthc Qual 2006; 28:37-44.

28. Rycroft-Malone J. Ingredients for change: revisiting a conceptual framework. Qual Saf Health Care 2002; 11:174-80.

29. Jenal S, Évora YDM. Revisão de literatura: implantação de prontuário eletrônico do paciente. J Health Inform 2012; 4:176-81.

30. Canêo PK, Rondina JM. Prontuário eletrônico do paciente: conhecendo as experiências de sua implantação. J Health Inform 2014; 6: 67-71.

31. Bhattacherjee A, Hikmet N. Physicians' resistance toward healthcare information technology: a theoretical model and empirical test. Eur J Inf Syst 2007; 16:725-37.

32. Klein KJ, Conn AB, Sorra JS. Implementing computerized technology: an organizational analysis. J Appl Psychol 2001; 86:811-24.

33. Tulu B, Horan TA, Burkhard R. Dimensions of work practice compatibility and influences on actual system use: examining physician use of online disability evaluation systems. In: Proceedings of Americas Conference on Information Systems. Omaha: Americas for Information Systems; 2005. p. 10-4.

34. Freire SM. Sigilo das informações. In: Agência Nacional de Saúde Suplementar; Ministério da Saúde, organizadores. Regulação \& saúde. v. 3. Brasília: Ministério da Saúde; 2004. p. 337-80. 
35. Campion-Awwad O, Hayton A, Smith L, Vuaran M. The National Programme for IT in the NHS: a case history. https://www.cl.cam. ac.uk/ rja14/Papers/npfit-mpp-2014-casehistory.pdf (acessado em 16/Dez/2015).

36. Pisa IT, Lopes PRL, Holanda AJ, Pires DF, Ruiz EES. MIDster: sistema distribuído de imagens médicas baseado em modelos peer-to-peer (P2P) e serviços web. In: Anais do IX Congresso Brasileiro de Informática em Saúde. http:// telemedicina.unifesp.br/pub/SBIS/CBIS2004/ trabalhos/livro.pdf (acessado em 30/Nov/2017).

37. Integrating Healthcare Enterprise. A peer to peer agent coordination framework for IHE based cross-community health record exchange. http://doc.rero.ch/record/209308/ files/A_Peer_to_Peer_Agent_Coordination_ Framework_for_IHE.pdf (acessado em 05/ Jan/2016).

38. Nardon FB, Furuie S, Tachinardi U. Novas tecnologias para construção do prontuário eletrônico do paciente. In: Anais do Congresso da Sociedade Brasileira de Informática em Saúde. http://www.tridedalo.com.br/fabiane/publica tions/NovasTecnologiasPEP.pdf (acessado em 30/Nov/2017).

39. Barros JV. Sistemas de informação e avaliação de desempenho hospitalar: a integração e interoperabilidade entre fontes de dados hospitalares [Dissertação de Mestrado]. São Paulo: Faculdade de Medicina, Universidade de São Paulo; 2008.

40. Neto JSA, Oliveira HJQ. Modelo de banco de dados distribuído para o prontuário eletrônico único do paciente. In: XXIV Congresso Brasileiro de Engenharia Biomédica. http://www.canal6.com. br/cbeb/2014/artigos/cbeb2014_submis sion_318.pdf (acessado em 06/Jun/2016).
41. Panitz LM. Registro eletrônico de saúde e produção de informações da atenção à saúde no SUS [Dissertação de Mestrado]. Rio de Janeiro: Escola Nacional de Saúde Pública Sergio Arouca, Fundação Oswaldo Cruz; 2014.

42. Portal Brasil. SUS usará supercomputadores para integrar prontuários eletrônicos de todo o País. http://www.brasil.gov.br/sau de/2016/12/sus-usara-supercomputadores -para-integrar-prontuarios-eletronicos-de-to do-o-pais (acessado em 30/Nov/2017).

43. Smith-Hillman AV. The implementation of electronic health records in Brazil: impact assessment. Rio de Janeiro: Agência Nacional de Saúde Suplementar; 2013.

44. Heeks R. Health information systems: failure, success and improvisation. Int J Med Inform 2006; 75:125-37.

45. Ministério da Saúde. Portaria no 589, de 20 de maio de 2015. Institui a Política Nacional de Informação e Informática em Saúde (PNIIS). Diário Oficial da União 2015; 22 maio.

46. Ministério da Saúde. Estratégia e-saúde para o Brasil. http://cspace.eportuguese.org/tiki-do wnload_file.php?fileId=1173 (acessado em 30/ Nov/2017). 


\section{Abstract}

The design and deployment of complex technologies like the electronic health record (EHR) involve technical, personal, social, and organizational issues. The Brazilian public and private scenario includes different local and regional initiatives for implementation of the electronic health record. The Brazilian Ministry of Health also has a proposal to develop a national EHR. The current study aimed to provide a comprehensive view of perceptions by health system administrators, professionals, and users concerning their experiences with the electronic health record and their opinions of the possibility of developing a national EHR. This qualitative study involved 28 semi-structured interviews. The results revealed both the diversity of factors that can influence the implementation of an electronic health record and the existence of convergences and aspects that tend to be valued differently according to the different points of view. Key aspects include discussions on the electronic health record's attributes and it impact on healthcare, especially in the case of local electronic health records, concerns over costs and confidentiality and privacy pertaining to electronic health records in general, and the possible implications of centralized versus decentralized data storage in the case of a national EHR. The interviews clearly showed the need to establish more effective communication among the various stakeholders, and that the different perspectives should be considered when drafting and deploying an EHR at the local, regional, and national levels.

Electronic Health Records; Public Health Informatics; Health Communication

\section{Resumen}

La concepción y la implementación de tecnologías complejas, como el registro electrónico de salud (RES), involucran tanto aspectos técnicos como cuestiones personales, sociales y organizacionales. Hay un escenario brasileño público y privado, diferentes iniciativas locales y regionales de implementación de RES. Existe incluso, por parte del Ministerio de Salud, la propuesta de conformación de un RES nacional. El estudio aquí presentado buscó propiciar una visión amplia de las percepciones de gestores, profesionales y usuarios del sistema de salud acerca de sus experiencias con el RES y sus opiniones acerca de la perspectiva de conformación de un RES nacional. Se trata de un estudio cualitativo que implicó la realización de 28 entrevistas semiestructuradas. Los resultados obtenidos explicitaron, además de la diversidad de factores que pueden influenciar la implementación del RES, la existencia de confluencias y de aspectos que tienden a ser valorados de modo distinto, conforme los diferentes puntos de vista. Entre los aspectos clave destacados en las percepciones de los entrevistados, vale enfatizar las discusiones acerca de atributos y del impacto del RES en el cuidado de salud, especialmente en el caso de los RES de uso local; las preocupaciones con los costes y con el sigilo y privacidad, relativos a los RES en general; $y$ las posibles implicaciones derivadas de la opción por la centralización o descentralización del almacenamiento de la información, en el caso del RES nacional. Quedó patente en las intervenciones de los entrevistados la necesidad de que sea establecida una comunicación más efectiva entre las partes interesadas, y que las diferentes perspectivas sean consideradas en la formulación e implementación de RES en los niveles local, regional y nacional.

Registros Electrónicos de Salud; Informática en Salud Pública; Comunicación en Salud
Recebido em 30/Out/2016

Versão final reapresentada em 29/Mai/2017 Aprovado em 13/Jun/2017 